Pacific Journal of Mathematics

BEHAVIOR OF GREEN LINES AT THE KURAMOCHI 


\title{
BEHAVIOR OF GREEN LINES AT THE KURAMOCHI BOUNDARY OF A RIEMANN SURFACE
}

\author{
Mitsuru Nakai and Leo Sario
}

We shall establish necessary and sufficient conditions, in terms of Green lines, for a point of the Kuramochi boundary $\Gamma^{k}$ of a hyperbolic Riemann surface $R$ to be of positive harmonic measure.

Explicitly, let $\mathfrak{B}$ be the bundle of all Green lines $l$ issuing from a fixed point of $R$. It forms a measure space with the Green measure. We call a subset $\mathfrak{A}$ of $\mathfrak{B}$ a distinguished bundle if it has positive measure and there exists a point $p$ in $\Gamma^{k}$ such that almost every $l$ in $\mathscr{U}$ terminates at $p$. The point $p$ will be referred to as the end of $\mathfrak{X}$.

Our main result is that a point $p$ of $\Gamma^{k}$ has positive measure if and only if there exists a distinguished bundle $\mathfrak{A}$ whose end is $p$.

We shall also give an intrinsic characterization of the latter property, without reference to points of $\Gamma^{k}$ : A bundle $\mathfrak{A}$ is distinguished if and only if it has positive measure and for every $H D$-function $u$ there exists a real number $c_{u}$ such that $u$ has the limit $c_{u}$ along almost every $l$ in $\mathfrak{U}$.

\section{Green lines}

1. Let $R$ be a hyperbolic Riemann surface, the hyperbolicity characterized by the existence of Green's functions. Fix a point $z_{0} \in R$ and denote by $g(z)=g\left(z, z_{0}\right)$ the Green's function on $R$ with singularity $z_{0}$. Consider the differential equations

$$
\begin{gathered}
\frac{d r(z)}{r(z)}=-d g(z), \quad r\left(z_{0}\right)=0, \\
d \theta(z)=-* d g(z) .
\end{gathered}
$$

Equation (1) has the unique solution $r(z)=e^{-g(z)}$ on $R$ with $0 \leqq r(z)<1$. In any simply connected subregion of $R-z_{0}$ where $d g(z) \neq 0$, equation (2) also has a solution $\theta(z)$, unique up to an additive constant. The global solution $\theta(z)$, however, is a multivalued harmonic function.

Set $G_{\rho}=\{z \in R \mid r(z)<\rho\}, C_{\rho}=\partial G_{\rho}(0<\rho<1)$. For a sufficiently small $\rho$, the analytic function $w=\varphi(z)=r(z) e^{i \theta(z)}$ is single-valued and gives a univalent conformal mapping of $G_{\rho}$ onto the disk $|w|<\rho$. Denote by $\rho_{0}$ the supremum of all $\rho$ with this property.

2. An open arc $\alpha$ is called a Green arc if $d g(z) \neq 0$ for all $z \in \alpha$, 
and a branch of $\theta$ is constant on $\alpha$. The set of Green arcs is partially ordered by inclusion. A maximal Green arc in this partially ordered set is called a Green line.

A Green line $l$ is said to issue from $z_{0}$ if $z_{0} \in \bar{l}$. We denote by $\mathfrak{B}$ the set of Green lines issuing from $z_{0}$ and use the suggestive term bundle for a subset $\mathfrak{A}$ of $\mathfrak{R}$, with the case $\mathfrak{A}=\mathfrak{B}$ not excluded.

For a fixed $\rho \in\left(0, \rho_{0}\right)$ and a given $p \in C_{\rho}$ let $l(p)$ be the Green line in $\mathfrak{B}$ passing through $p$. Making use of the function $w=\varphi(z)=$ $r(z) e^{i \theta(z)}$ we see that the mapping $p \rightarrow l(p)$ is bijective; let $p(l)$ be the inverse mapping. We call a bundle $\mathfrak{U} \subset \mathfrak{B}$ measurable if $p(\mathfrak{H})$ is measurable in $C_{\rho}$, and define the Green measure of $\mathfrak{A}$ by

$$
m(\mathfrak{U})=\frac{1}{2 \pi} \int_{p(\mathfrak{X})} d \theta(z)=-\frac{1}{2 \pi} \int_{p(\mathfrak{Q})} * d g(z) .
$$

The space $(\mathfrak{B}, m)$ is a probability space, i.e., a measure space of total measure unity. The definition is independent of the choice of $\rho \in\left(0, \rho_{0}\right)$.

3. Fix an $l \in \mathfrak{B}$. The number $a(l)=\sup _{z \in l} r(z)$ is in $(0,1]$. If $a(l)<1$, then $l$ terminates at a point of $R$ at which $d g=0$. Such an $l$ is called singular. If $a(l)=1$, then $l$ tends to the ideal boundary of $R$ and is called regular. The bundle $\mathfrak{B}_{r}$ of regular Green lines "almost" comprises $\mathfrak{B}$, that is, $m\left(\mathfrak{B}_{r}\right)=1$. This is a result of BrelotChoquet [1] (cf. [7], [8]).

\section{Compactifications.}

4. Let $R^{c}$ be a compactification of $R$, i.e., a compact Hausdorff space containing $R$ as its open dense subspace. For a bounded continuous function $\varphi$ on the ideal boundary $\Gamma^{c}=R^{c}-R$ of $R$, denote by $U_{\varphi}^{R^{c}}$ the class of superharmonic functions $s$ on $R$ such that

$$
\lim _{z \in R, z \rightarrow p} \inf _{s(z) \geqq \varphi(p)}
$$

for every $p \in \Gamma^{c}$. The function

$$
H_{\varphi}^{R^{c}}(z)=\inf _{s \in U_{\varphi}^{R^{c}}} s(z)
$$

is harmonic on $R$. We assume that $R^{c}$ is a resolutive compactification (cf. Constantinescu-Cornea [2]), that is, $\varphi \rightarrow H_{\varphi}^{R^{c}}(z)$ is a continuous linear functional. Then for $z_{0} \in R$ there exists a measure $\mu^{c}$, called the harmonic measure on $\Gamma^{\circ}$, and a function $P^{c}(z, p)$ on $R \times \Gamma^{c}$ with properties $P_{c}\left(z_{0}, p\right) \equiv 1$,

$$
H_{\varphi}^{R^{\bullet}}(z)=\int_{\Gamma^{c}} P^{c}(z, p) \varphi(p) d \mu^{c}(p) .
$$


This representation extends to bounded Borel measurable functions $\varphi$ on $\Gamma^{c}$.

Let $\widetilde{H D}(R)$ be the class of harmonic functions $u \geqq 0$ on $R$ such that there exists a decreasing sequence $\left\{u_{n}\right\} \subset H D(R)$ with $u=\lim _{n} u_{n}$ on $R$. A function $u \in \widetilde{H D}(R)$ is said to be $\widetilde{H D}$-minimal if for every $v \in \widetilde{H D}(R)$ with $v \leqq u$ on $R$ there exists a constant $c_{v}$ such that $v=c_{v} u$ on $R$. We shall call the compactification $R^{c} \widetilde{H D}$-compatible if the following condition is satisfied: $u \in \widetilde{H D}(R)$ is $\widetilde{H D}$-minimal if and only if there exists a point $p_{0} \in \Gamma^{c}$ with $\mu^{c}\left(p_{0}\right)>0$ and a number $k>0$ such that

$$
u(z)=k \int_{p_{0}} P^{c}(z, p) d \mu^{c}(p) .
$$

5. The Royden compactification $R^{*}$ of $R$, with the Royden boundary $\Gamma=R^{*}-R$, is a typical example of an $\widetilde{H D}$-compatible compactification (see [6], [8]). We let $\mu$ and $P$ stand for $\mu^{c}$ and $P^{c}$ corresponding to $R^{*}$.

A compactification $R^{c}$ is said to lie below $R^{*}$ if there exists a continuous mapping $\pi=\pi^{c}$ of $R^{*}$ onto $R^{c}$ such that $\pi \mid R$ is the identity and $\pi^{-1}(R)=R$. Clearly $\pi$ is unique and we have

$$
\int_{\Gamma^{c}} P^{c}(z, p) \varphi(p) d \mu^{c}(p)=\int_{\Gamma} P\left(z, p^{*}\right) \varphi\left(\pi\left(p^{*}\right)\right) d \mu\left(p^{*}\right)
$$

for every bounded Borel function $\varphi$ on $\Gamma^{c}$.

6. We are interested in the behavior of $l \in \mathfrak{B}_{r}$ in $R^{c}$. We set

$$
e^{c}(l)=\bar{l}^{c}-l \cup\left\{z_{0}\right\},
$$

with $\bar{l}^{c}$ the closure of $l$ in $R^{c}$, and call $e^{c}(l)$ the end part of $l$ in $R^{c}$. It is a compact set in $\Gamma^{c}$. If

$$
\mathfrak{B}^{c}=\left\{l \in \mathfrak{B}_{r} \mid e^{c}(l) \text { is a single point }\right\}
$$

is of measure $m\left(\mathfrak{B}^{c}\right)=1$, then we call $R^{c}$ Green-compatible.

We shall make use of a result of Maeda [4]: A metrizable compactification $R^{c}$ which lies below $R^{*}$ is Green-compatible.

7. A compactification $R^{c}$ of $R$ is said to be of type $G$ if $R^{c}$ is metrizable, $\widetilde{H D}$-compatible, and lies below $R^{*}$. Note that $R^{c}$ is then Green-compatible. An important example:

Proposition. The Kuramochi compactification $R^{k}$ of $R$ is of type $G$.

In fact, metrizability and $\widetilde{H D}$-compatibility of $R^{k}$ are immediate 
consequences of related results of Constantinescu-Cornea [2, pp. 171 and 169]. That $R^{k}$ lies below $R^{*}$ follows from the definition of the Kuramochi compactification given in [2, p. 167].

$R^{k}$ is actually the only significant compactification of type $G$ known thus far. For a general discussion of its properties we also refer to [5].

\section{Distinguished bundles.}

8. Let $R^{c}$ be a compactification of $R$ of type $G$. We call a bundle $\mathfrak{Y} \subset \mathfrak{B} R^{c}$-distinguished if $m(\mathfrak{H})>0$ and there exists a point $p \in \Gamma^{c}$ such that $e^{c}(l)=p$ for almost every $l \in \mathfrak{U}$. The point $p$ will be referred to as the end of $\mathfrak{U}$. In the case $R^{c}=R^{k}$ we simply say that $\mathfrak{U}$ is distinguished.

We shall characterize points $p \in \Gamma^{\circ}$ of positive measure in terms of $R^{c}$-distinguished bundles:

THEOREM. Let $R^{c}$ be a compactification of type $G$ of a hyperbolic Riemann surface $R$. A point $p \in \Gamma^{c}=R^{c}-R$ has positive harmonic measure if and only if there exists an $R^{c}$-distinguished bundle $\mathfrak{X}$ with end $p$.

The proof will be given in $9-13$.

9. Let $\Gamma=R^{*}-R$ be the Royden boundary of $R$. For $l \in \mathfrak{B}_{r}$ denote by $e(l)$ the set $\bar{l}-l \cup\left\{z_{0}\right\}$ in $\Gamma$, with $\bar{l}$ the closure of $l$ in $R^{*}$. Given a subset $S \subset \Gamma$ we write

$$
\widetilde{S}=\{l \in \mathfrak{B} \mid e(l) \cap S \neq \varnothing\}, \quad \check{S}=\{l \in \mathfrak{B} \mid e(l) \subset S\} .
$$

We shall employ the following auxiliary result ([7], [8]): For every $F_{\sigma}$-set $K$ (resp. $G_{\delta}$-set $U$ ) in $\Gamma$

$$
\bar{m}(\widetilde{K}) \leqq \mu(K), \quad \underline{m}(\check{U}) \geqq \mu(U),
$$

where $\bar{m}$ and $\underline{m}$ are the outer and inner measures induced by $m$.

Let $p^{*}$ be on the Royden harmonic boundary $\Delta$ of $R$. The set

$$
\Lambda_{p^{*}}=\left\{q^{*} \in \Gamma \mid u\left(q^{*}\right)=u\left(p^{*}\right) \text { for all } u \in H B D(R)\right\}
$$

is called a block at $p^{*}$. It is known ([7], [8]) that it has a measurable $\tilde{A}_{p^{*}}$,

$$
m\left(\tilde{\Lambda}_{p^{*}}\right)=\mu\left(p^{*}\right),
$$

and that

$$
u\left(p^{*}\right)=\lim _{z \in l, r(z) \rightarrow 1} u(z)
$$

for every $u \in H D(R)$ and almost every $l \in \tilde{A}_{p^{*}}$. 
10. Suppose $\mathfrak{A}$ is an $R^{c}$-distinguished bundle with end $p \in \Gamma^{c}$. We are to prove that $\mu^{c}(p)>0$. Take the projection $\pi=\pi^{c}$ of $R^{*}$ onto $R^{c}$ (see 5). The set $K=\pi^{-1}(p)$ is compact and clearly $\mathfrak{A} \subset \widetilde{K}$. By (9),

$$
0<m(\mathfrak{A}) \leqq \bar{m}(\widetilde{K}) \leqq \mu(K) .
$$

From (6) it follows that $\mu(K)=\mu\left(\pi^{-1}(p)\right)=\pi^{c}(p)$. Therefore

$$
0<m(\mathfrak{U}) \leqq \mu^{c}(p)
$$

11. Conversely suppose that $p \in \Gamma^{c}$ and $\mu^{c}(p)>0$. Since $R^{c}$ is $\widetilde{H D}$-compatible, the function $u(z)=\int_{p} P^{c}(z, q) d \mu^{c}(q)$ is $\widetilde{H D}$-minimal on $R$. By (6) we see that

$$
u(z)=\int_{\pi^{-1}(p)} P\left(z, q^{*}\right) d \mu\left(q^{*}\right) .
$$

Since $R^{*}$ is also $\widetilde{H D}$-compatible and the integral representation (12) of the $\widetilde{H D}$-function $u$ is unique up to a boundary function vanishing $\mu$-almost everywhere on $\Gamma([6],[8])$, we conclude that there exists a point $p^{*} \in \pi^{-1}(p)$ with $\mu\left(p^{*}\right)=\mu\left(\pi^{-1}(p)\right)>0$. Observe that

$$
m\left(\widetilde{\Lambda}_{p^{*}}\right)=\mu\left(p^{*}\right)>0 .
$$

In view of the Green-compatibility of $R^{c}$, there exists a measurable subset $\mathfrak{A} \subset \widetilde{\Lambda}_{p^{*}}$ with $m\left(\widetilde{\Lambda}_{p^{*}}\right)=m(\mathfrak{U})$ and such that $e^{c}(l)$ is a single point in $\Gamma^{c}$ for each $l \in \mathfrak{A}$.

To conclude that $\mathfrak{A}$ is an $R^{c}$-distinguished bundle with end $p$, we must show that $\mathfrak{X}^{\prime}=\left\{l \in \mathfrak{A} \mid e^{c}(l) \neq p\right\}$ is of $m$-measure zero. For this purpose take a sequence $\left\{U_{n}\right\}_{1}^{\infty}$ of open sets in $\Gamma^{c}$ with

$$
U_{n+1} \subset \bar{U}_{n+1} \subset U_{n}, \quad \bigcap_{1}^{\infty} U_{n}=\{p\} .
$$

Let $\mathfrak{A}_{n}^{\prime}=\left\{l \in \mathfrak{X}^{\prime} \mid e^{c}(l) \notin U_{n}\right\}$. Since $\mathfrak{U}^{\prime}=\bigcup_{n=1}^{\infty} \mathfrak{A}_{n}^{\prime}$, it suffices to show that $m\left(\mathfrak{Y}_{n}^{\prime}\right)=0$ for every $n$.

12. First we assume that $R \notin O_{H D}$. For an arbitrarily fixed $n$ there exists a $u_{n} \in H B D(R)$ such that

$$
0 \leqq u_{n}\left|\Delta \leqq 1, u_{n}\right| \pi^{-1}\left(U_{n+1}\right) \cap \Delta=1, u_{n} \mid\left(\Delta-\pi^{-1}\left(U_{n}\right)\right)=0 .
$$

In view of (11), there exists a measurable subset $\mathfrak{U}_{n}^{\prime \prime} \subset \mathfrak{U}_{n}^{\prime}$ with $m\left(\mathfrak{H}_{n}^{\prime}-\mathfrak{I}_{n}^{\prime \prime}\right)=0$ and

$$
1=u_{n}\left(p^{*}\right)=\lim _{z \in l, r(z) \rightarrow 1} u_{n}(z)
$$

for every $l \in \mathfrak{A}_{n}^{\prime \prime}$. The set $E_{n}=\left\{q^{*} \in \Gamma \mid u_{n}\left(q^{*}\right)<\frac{1}{2}\right\}$ is open in $\Gamma^{r}$. By 
(15), $e(l) \cap E_{n}=\varnothing$ for every $l \in \mathfrak{Y}_{n}^{\prime \prime}$. Because of the definition of $\mathfrak{Y}_{n}^{\prime}$, it is also clear that $e(l) \cap \pi^{-1}\left(U_{n}\right)=\varnothing$ for every $l \in \mathfrak{U}_{n}^{\prime \prime}$. Since the set $K_{n}=\Gamma-\pi^{-1}\left(U_{n}\right) \cup E_{n}$ is compact and $\pi^{-1}\left(U_{n}\right) \cup E_{n} \supset \Delta$, we have $K_{n} \subset$ $\Gamma-\Delta$ and a fortiori $\mu\left(K_{n}\right)=0$.

On the other hand, $e(l) \subset K_{n}$ for every $l \in \mathfrak{A}_{n}^{\prime \prime}$. Therefore $\mathfrak{A}_{n}^{\prime \prime} \subset$ $\check{K}_{n} \subset \widetilde{K}_{n}$. In view of (9), we obtain

$$
m\left(\mathfrak{U}_{n}^{\prime \prime}\right) \leqq \bar{m}\left(\widetilde{K}_{n}\right) \leqq \mu\left(K_{n}\right)=0
$$

and conclude that $m\left(\mathfrak{Y}_{n}^{\prime}\right)=m\left(\mathfrak{R}_{n}^{\prime \prime}\right)=0$.

13. If $R \in O_{H D}$, then $\Delta$ consists of a single point and consequently $\Delta=\left\{p^{*}\right\}$. The set $F_{n}=\Gamma-\pi^{-1}\left(U_{n}\right)$ is compact in $\Gamma-\Delta$ and hence $\mu\left(F_{n}\right)=0$. By the definition of $\mathfrak{A}_{n}^{\prime}$ we have $\mathfrak{U}_{n}^{\prime} \subset \breve{F}_{n} \subset \widetilde{F}_{n}$. Therefore $m\left(\mathfrak{A}_{n}^{\prime}\right) \leqq m\left(\widetilde{F}_{n}\right) \leqq \mu\left(F_{n}\right)=0$. The proof of Theorem 8 is herewith complete.

\section{Characterization of distinguished bundles.}

14. We next give necessary and sufficient conditions for a bundle to be distinguished, without referring to its end:

THEOREM. Let $R^{c}$ be a compactification of type $G$ of a hyperbolic Riemann surface $R . \quad A$ bundle $\mathfrak{A} \subset \mathfrak{B}$ is $R^{c}$-distinguished if and only if $m(\mathfrak{I})>0$ and for each $u \in H D(R)$ there exists a number $c_{u}$ such that

$$
\lim _{z \in l, r(z) \rightarrow 1} u(z)=c_{u}
$$

for almost every $l \in \mathfrak{A}$.

The proof will be given in 15-18.

15. First suppose $\mathfrak{A}$ is $R^{c}$-distinguished with end $p \in \Gamma^{c}$. Then by 10 and 11 , there exists a point $p^{*} \in K=\pi^{-1}(p)$ such that

$$
0<\mu^{c}(p)=\mu(K)=\mu\left(p^{*}\right) \text {. }
$$

Fix a $u \in H D(R)$. By the Godefroid theorem [3] (see also [7], [8]),

$$
u(l)=\lim _{z \in l, r(z) \rightarrow 1} u(z)
$$

exists for almost every $l \in \mathfrak{B}_{r}$. On omiting from $\mathfrak{A}$ a set of measure zero we may assume that $u(l)$ in (17) exists for every $l \in \mathfrak{A}$. We may also suppose that $e^{c}(l)=p$ and a fortiori $e(l) \subset K$ for every $l \in \mathfrak{A}$.

Since $\mu\left(p^{*}\right)>0,\left|u\left(p^{*}\right)\right|<\infty$ (cf. [6], [8]). Let

$$
\mathfrak{A}^{\prime}=\left\{l \in \mathfrak{A} \mid u(l)-u\left(p^{*}\right) \neq 0\right\}
$$


and

$$
K_{n}=\left\{q^{*} \in K|| u\left(q^{*}\right)-u\left(p^{*}\right) \mid \geqq 1 / n\right\} .
$$

Clearly $K_{n}$ is a compact set. For $l \in \mathfrak{A}^{\prime}$ and $q^{*} \in e(l)$, we have $u(l)=$ $u\left(q^{*}\right)$ by (17) and the continuity of $u$ on $R^{*}$. Therefore $\left|u\left(q^{*}\right)-u\left(p^{*}\right)\right| \geqq$ $1 / n$ for some $n$ and a fortiori $e(l) \subset K_{n}$. It follows that

$$
\mathfrak{U}^{\prime} \subset \bigcup_{n=1}^{\infty} \check{K}_{n} \subset \bigcup_{n=1}^{\infty} \widetilde{K}_{n},
$$

which by (9) gives

$$
m\left(\mathfrak{U}^{\prime}\right) \leqq \bar{m}\left(\bigcup_{n=1}^{\infty} \widetilde{K}_{n}\right) \leqq \sum_{n=1}^{\infty} \bar{m}\left(\widetilde{K}_{n}\right) \leqq \sum_{n=1}^{\infty} \mu\left(K_{n}\right) .
$$

From $K_{n} \subset K-p^{*}$ and $\mu(K)=\mu\left(p^{*}\right)$, we obtain $\mu\left(K_{n}\right)=0$. Consequently $m\left(\mathfrak{U}^{\prime}\right)=0$ and, since

$$
\lim _{z \in l, r(z) \rightarrow 1} u(z)=u(l)=u\left(p^{*}\right)
$$

for every $l \in \mathfrak{A}-\mathfrak{A}^{\prime}$, we have (16) for almost every $l \in \mathfrak{A}$.

16. Conversely suppose that, for a bundle $\mathfrak{A} \subset \mathfrak{B}$ with $m(\mathfrak{U})>0$, (16) is satisfied. We may assume that $e^{c}(l)$ is a single point in $\Gamma^{c}$ for every $l \in \mathfrak{A}$.

First consider the case $R \in O_{H D}$. The harmonic boundary $\Delta$ consists of a single point $p^{*}$ and $\mu\left(p^{*}\right)>0$. Let $p=\pi\left(p^{*}\right)$. Take a sequence $\left\{U_{n}\right\}_{1}^{\infty}$ of open sets in $\Gamma^{c}$ such that $\bar{U}_{n+1} \subset U_{n}$ and $\bigcap_{1}^{\infty} U_{n}=\{p\}$. For the bundles $\mathfrak{U}_{n}^{\prime}=\left\{l \in \mathfrak{A} \mid e^{c}(l) \notin U_{n}\right\}, n=1,2, \cdots$, and

$$
\mathfrak{A}^{\prime}=\left\{l \in \mathfrak{A} \mid e^{c}(l) \neq p\right\}
$$

we have $\mathfrak{X}^{\prime}=\bigcup_{1}^{\infty} \mathfrak{U}_{n}^{\prime}$. Set $K_{n}=\Gamma-\pi^{-1}\left(U_{n}\right) \subset \Gamma-\Delta$. Every $l \in \mathfrak{X}_{n}^{\prime}$ has $e(l) \subset K_{n}$ and we obtain $\mathfrak{U}_{n}^{\prime} \subset \breve{K}_{n} \subset \widetilde{K}_{n}$. Hence

$$
m\left(\mathfrak{A}_{n}^{\prime}\right) \leqq \bar{m}\left(\widetilde{K}_{n}\right) \leqq \mu\left(K_{n}\right)=0
$$

and therefore $m\left(\mathfrak{X}^{\prime}\right)=0$, i.e., $e^{c}(l)=p$ for almost every $l \in \mathfrak{A}$. This proves that $\mathfrak{A}$ is $R^{c}$-distinguished.

17. Next suppose $R \notin O_{H D}$. The family $T(\mathfrak{H})=\{u \in H B D(R) \mid 0 \leqq u \leqq 1$ on $R, u(l)=1$ for almost every $l \in \mathfrak{A}\}$ is a Perron family and

$$
s(z)=\inf \{u(z) \mid u \in T(\mathfrak{U})\}
$$

is an $\widetilde{H D}$-minimal function on $R$ (see [7], [8]). We can therefore choose a decreasing sequence $\left\{h_{n}\right\} \subset T(\mathfrak{H})$ such that 


$$
s(z)=\lim _{n} h_{n}(z)
$$

on $R$. Let $\mathfrak{2}_{0}$ be a measurables subset of $\mathfrak{U}$ with $m(\mathfrak{U})=m\left(\mathfrak{H}_{0}\right)$ such that $h_{n}(l)$ exists and equals unity for every $n=1,2, \cdots$, and ${ }_{2}^{F}$ every $l \in \mathfrak{A}_{0}$. We set

$$
\bar{s}(l)=\limsup _{z \in l, r(z) \rightarrow 1} s(z)
$$

and observe that

$$
s\left(z_{0}\right)=\int_{\mathfrak{B}} s\left(r e^{i l}\right) d m(l) \leqq \int_{\mathfrak{B}} h_{n}\left(r e^{i l}\right) d m(l)=h_{n}\left(z_{0}\right)
$$

for every $r \in(0,1)$ (see [7], [8]). By Fatou's lemma

$$
s\left(z_{0}\right) \leqq \int_{\mathfrak{B}} \bar{s}(l) d m(l) \leqq \int_{\mathfrak{B}} h_{n}(l) d m(l)=h_{n}\left(z_{0}\right) .
$$

Let $h(l)=\lim _{n} h_{n}(l)$. Since $h_{n}(l) \geqq \bar{s}(l)$ and

$$
0 \leqq \int_{\mathfrak{B}}(h(l)-\bar{s}(l)) d m(l) \leqq \lim _{n \rightarrow \infty}\left(h_{n}\left(z_{0}\right)-s\left(z_{0}\right)\right)=0,
$$

we conclude that $\bar{s}(l)=h(l)$ almost everywhere on $\mathfrak{B}$. In view of $h(l)=1$ for every $l \in \mathfrak{N}_{0}$ we may suppose that

$$
\bar{s}(l)=1 \quad(l \in \mathfrak{U}) \text {. }
$$

18. The remainder of the proof is analogous to that in 11-12. In fact, since $s$ is $\widetilde{H D}$-minimal, there exist points $p$ and $p^{*}$ in $\Gamma^{c}$ and $\Gamma$ respectively such that $\mu^{c}(p)=\mu\left(p^{*}\right)>0, p^{*} \in \pi^{-1}(p)$, and

$$
s(z)=\int_{p} P^{c}(z, q) d \mu^{c}(q)=\int_{p^{*}} P\left(z, q^{*}\right) d \mu\left(q^{*}\right) .
$$

We wish to show that $e^{c}(l)=p$ for almost every $l \in \mathfrak{U}$, that is, $\mathfrak{A}$ is $R^{c}$-distinguished with end $p$. For this purpose set $\mathfrak{Y}^{\prime}=\left\{l \in \mathfrak{N} \mid e^{c}(l) \neq p\right\}$. To see that $m\left(\mathfrak{Y}^{\prime}\right)=0$ take a sequence $\left\{U_{n}\right\}$ of open sets in $\Gamma^{c}$ such that

$$
\bar{U}_{n+1} \subset U_{n}, \quad \bigcap_{1}^{\infty} U_{n}=\{p\} .
$$

For $\mathfrak{A}_{n}^{\prime}=\left\{l \in \mathfrak{A} \mid e^{c}(l) \notin U_{n}\right\}$ we have $\mathfrak{H}=\bigcup_{1}^{\infty} \mathfrak{V}_{n}^{\prime}$ and it suffices to show that $m\left(\mathfrak{U}_{n}^{\prime}\right)=0$ for every $n=1,2, \cdots$. Take a function $u_{n} \in H B D(R)$ with

$$
0 \leqq u_{n}\left|\Delta \leqq 1, u_{n}\right| \pi^{-1}\left(U_{n+1}\right) \cap \Delta=1, u_{n} \mid\left(\Delta-\pi^{-1}\left(U_{n}\right)\right)=0 .
$$

We may suppose $u_{n}(l)$ exists for every $l \in \mathfrak{T}$. Since $1 \geqq u_{n} \geqq s$ on $R$, (20) implies that

$$
u_{n}(l)=1 \quad(l \in \mathfrak{X})
$$


Clearly $e(l) \subset \Gamma-\pi^{-1}\left(U_{n}\right)$ for every $l \in \mathfrak{P}_{n}^{\prime}$. Moreover, if we set $E_{n}=$ $\left\{q^{*} \in \Gamma \mid u_{n}\left(q^{*}\right)<\frac{1}{2}\right\}$, then $e(l) \subset \Gamma-E_{n} \cup \pi^{-1}\left(U_{n}\right)=K_{n}$ for every $l \in \mathfrak{P I}_{n}^{\prime}$. Since $K_{n}$ is compact and contained in $\Gamma-\Delta$,

$$
\mathfrak{V}_{n}^{\prime} \subset \check{K}_{n} \subset \widetilde{K}_{n}
$$

implies that

$$
\left.m(\mathfrak{Z}\}_{n}^{\prime}\right) \leqq \bar{m}\left(\widetilde{K}_{n}\right)=\mu\left(K_{n}\right)=0 \text {. }
$$

The proof of Theorem 14 is herewith complete.

\section{Conclusion.}

19. Recall that a bundle $\mathfrak{U} \subset \mathfrak{B}$ is distinguished with end $p$ on the Kuramochi boundary if $m(\mathfrak{i})>0$ and almost every Green line in $\mathfrak{U}$ terminates at $p$. Since the Kuramochi compactification is of type $G$, Theorems 8 and 14 imply:

THeOREM. A point $p$ of the Kuramochi boundary of a hyperbolic Riemann surface $R$ has positive measure if and only if there exists a distinguished bundle $\mathfrak{i}$ of Green lines with end $p$.

$A$ bundle $\mathfrak{X}$ of Green lines with $m(\mathfrak{H})>0$ is distinguished if and only if, for every $u \in H D(R)$, there exists a number $c_{u}$ such that the "radial limit" $\lim _{z \in l, r(z) \rightarrow 1} u(z)$ exists and equals $c_{u}$ for almot every $l \in \mathfrak{T}$.

\section{REFERENCES}

1. M. Brelot and G. Choquet, Espace et lignes de Green, Ann. Inst. Fourier 3 (1952), 199-263.

2. C. Constantinescu and A. Cornea, Ideale Ränder Riemannscher Flächen, SpringerVerlag, 1963.

3. M. Godefroid, Une propriété des fonctions BLD dans un espace de Green, Ann. Inst. Fourier 9 (1959), 301-304.

4. F.-Y. Maeda, Notes on Green lines and Kuramochi boundary of a Green space, J. Sci. Hiroshima Univ. 28 (1964), 59-66.

5. F.-Y. Maeda, M. Ohtsuka, et al., Kuramochi Boundaries of Riemann Surfaces, Lecture Notes 58, Springer-Verlag, 1968.

6. M. Nakai, A measure on the harmonic boundary of a Riemann surface, Nagoya Math. J. 17 (1960), 181-218.

7. Behavior of Green lines at Royden's boundary of Riemann surfaces, Nagoya Math. J. 24 (1964), 1-27.

8. L. Sario and M. Nakai, Classification Theory of Riemann Surfaces, SpringerVerlag, 1970.

Received April 13, 1970. The work was sponsored by the U.S. Army Research Office-Durham, Grant DA-AROD-31-124-G855, University of California, Los Angeles.

NAGOYA UNIVERSITY

ChIKUSA-KU, NAGOYA, JAPAN

UNIVERSITY OF CALIFORNIA

Los ANgeles, CALIFornia 



\section{PACIFIC JOURNAL OF MATHEMATICS}

\section{EDITORS}

H. SAMELson

Stanford University

Stanford, California 94305

R. R. PHelPS

University of Washington

Seattle, Washington 98105
J. Dugundu

Department of Mathematics

University of Southern Californle

Los Angeles, California 9.0007

RICHARD ARENS

University of California

Los Angeles, California 9.0024

\section{ASSOCIATE EDITORS}
E. F. BECKENBACH
B. H. NeumanN
F. WOLE
K. Yoshida

\section{SUPPORTING INSTITUTIONS}

UNIVERSITY OF BRITISH COLUMBIA

CALIFORNIA INSTITUTE OF TECHNOLOGY

UNIVERSITY OF CALIFORNIA

MONTANA STATE UNIVERSITY

UNIVERSITY OF NEVADA

NEW MEXICO STATE UNIVERSITY

OREGON STATE UNIVERSITY

UNIVERSITY OF OREGON

OSAKA UNIVERSITY

UNIVERSITY OF SOUTHERN CALIFORNIA

\author{
STANFORD UNIVERSITY \\ UNIVERSITY OF TOKYO \\ UNIVERSITY OF UTAH \\ WASHINGTON STATE UNIVERSITY \\ UNIVERSITY OF WASHINGTON \\ AMERICAN MATHEMATICAL SOCIETY \\ CHEVRON RESEARCH CORPORATION \\ NAVAL WEAPONS CENTER
}

The Supporting Institutions listed above contribute to the cost of publication of this Journal, but they are not owners or publishers and have no responsibility for its content or policies.

Mathematical papers intended for publication in the Pacific Journal of Mathematics should be in typed form or offset-reproduced, (not dittoed), double spaced with large margins. Underline Greek letters in red, German in green, and script in blue. The first paragraph or two must be capable of being used separately as a synopsis of the entire paper. The editorial "we" must not be used in the synopsis, and items of the bibliography should not be cited there unless absolutely necessary, in which case they must be identified by author and Journal, rather than by item number. Manuscripts, in duplicate if possible, may be sent to any one of the four editors. Please classify according to the scheme of Math. Rev. Index to Vol. 39. All other communications to the editors should be addressed to the managing editor, Richard Arens, University of California, Los Angeles, California, 90024.

50 reprints are provided free for each article; additional copies may be obtained at cost in multiples of 50 .

The Pacific Journal of Mathematics is published monthly. Effective with Volume 16 the price per volume (3 numbers) is $\$ 8.00$; single issues, $\$ 3.00$. Special price for current issues to individual faculty members of supporting institutions and to individual members of the American Mathematical Society: $\$ 4.00$ per volume; single issues $\$ 1.50$. Back numbers are available.

Subscriptions, orders for back numbers, and changes of address should be sent to Pacific Journal of Mathematics, 103 Highland Boulevard, Berkeley, California, 94708.

PUBLISHED BY PACIFIC JOURNAL OF MATHEMATICS, A NON-PROFIT CORPORATION

Printed at Kokusai Bunken Insatsusha (International Academic Printing Co., Ltd.), 7-17, Fujimi 2-chome, Chiyoda-ku, Tokyo, Japan. 


\section{Pacific Journal of Mathematics}

\section{Vol. 36, No. 2 December, 1971}

George E. Andrews, On a partition problem of H. L. Alder ............ 279

Thomas Craig Brown, An interesting combinatorial method in the theory of locally finite semigroups .......................... 285

Yuen-Kwok Chan, A constructive proof of Sard's theorem ............. 291

Charles Vernon Coffman, Spectral theory of monotone Hammerstein

operators...................................... 303

Edward Dewey Davis, Regular sequences and minimal bases .......... 323

Israel (Yitzchak) Nathan Herstein and Lance W. Small, Regular elements in

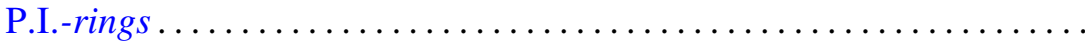

Marcel Herzog, Intersections of nilpotent Hall subgroups ..............

W. N. Hudson, Volterra transformations of the Wiener measure on the space

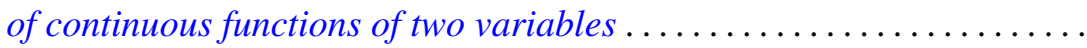

J. H. V. Hunt, An n-arc theorem for Peano spaces ................ 351

Arnold Joseph Insel, A decomposition theorem for topological group

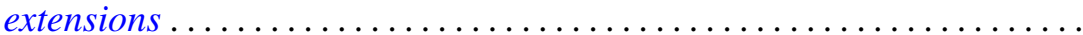

Caulton Lee Irwin, Inverting operators for singular boundary value

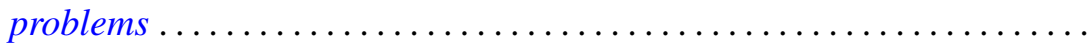

Abraham A. Klein, Matrix rings of finite degree of nilpotency ............ 387

Wei-Eihn Kuan, On the hyperplane section through a rational point of an algebraic variety...

John Hathway Lindsey, II, On a six-dimensional projective representation of $\mathrm{PSU}_{4}(3)$

Jorge Martinez, Approximation by archimedean lattice cones ...

J. F. McClendon, On stable fiber space obstructions .........

Mitsuru Nakai and Leo Sario, Behavior of Green lines at the Kuramochi boundary of a Riemann surface ....................

Donald Steven Passman, Linear identities in group rings. I. .

Donald Steven Passman, Linear identities in group rings. II ...

David S. Promislow, The Kakutani theorem for tensor products of

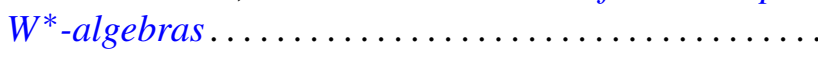

Richard Lewis Roth, On the conjugating representation of a finite group

Bert Alan Taylor, On weighted polynomial approximation of entire functions...

William Charles Waterhouse, Divisor classes in pseudo Galois

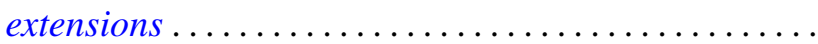

Chi Song Wong, Subadditive functions ...

Ta-Sun $\mathrm{Wu}$, A note on the minimality of certain bitransformation groups 